LA-UR $-87-3703$

$\operatorname{CONF}-8702100--14$

$\therefore .10$.

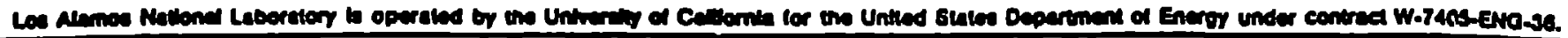

LA-UR- $-87-3703$

DE88 003179

IIIE: RELATIVISTIC HYDRODYNAMICS AND HEAVY ION REACTIONS

Aunorap Dantel Strottwan, T-2

suBmited TO: Proceedings of the Los Alamos/Leme11 Conference on Mathematics and Numerical Methods, Edited by R. Alcouffe, D. Holm, and P. J. O'Rourke, February 2-6, 1987. 


\title{
RELATIVISTIC HYDRODYNAMICS AND HEAVY ION REACTIONS
}

\author{
D. Strottman \\ Theoretical Division \\ Los Alamos National Laboratory \\ Los Alamos, NM 87544
}

To be published in the Proceedings of the Los Alamos/Lemeil Conference on Mathematics and Numerical Methods, edited by R. Alcouffe, D. Holm and P.J. O'Rourke

\section{DISC L.AIMER}

\begin{abstract}
This report w is prepured as an account of work spunsored by an apency of the l/nited Sithes fiovernment Neither the Inited Situtes (ioverument nur any agency thereof, nor any of theit comployees, makes uny warrant, express or implieci, ur asoumes any legal liability of responsihili,y for the accuracy, completeness, or usefulaes., of any information, appuratus, prexfuct, or proxess diw:lised, or represents ihal its use would not infrunge pr valely owned rights Kefer ence herein to any specific commerciul product, proxess, "re service by trade name. trademurk.

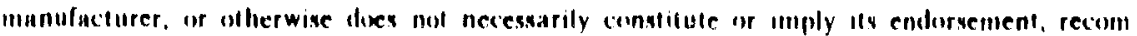

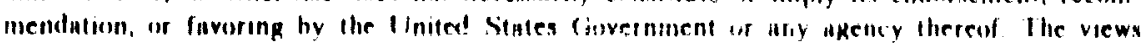
and opinions of nuthers expresseal herein du mot necessurily seute or reflect those at the IInited Silutes Cinvernment or any ngency heteot
\end{abstract}




\title{
RELATIVISTI ${ }^{\sim}$.IYDRODYNAMICS AND HEAVY ION REACTIONS
}

\author{
D. Strottman \\ Theoretical Division \\ Los Alamos National Laboratory \\ Los Alamos, NM 87545
}

\begin{abstract}
Abstracs
The possibility of forming a new state of nuclear matter - the quarkgluon plasma - in relativistic heavy ion collisions is currently the subject of intense theoretical and experimental investigation. The possible formation and evolution of the plasma is investigated using relativistic hydrodynamics. Nuclei are nearly transparent at the high bombirding energies involved and it is necessary to use a two-nluid model to describe the initial stages of the reaction. The current status of the numerical techniques and the relevant physics is reviewed.
\end{abstract}

It is generally conceded that nucleons are composed of quarks and gluons, although these basic constituents of matter have not been directly observed. The quarks are (massless) point-like objects carrying charge. baryon number and other quantum numbers such as strangeness, color and flavor. The gluons are the massless agents which mediate the strong interaction. The quark-quark interaction increases with their relative distance and thus are normally confined to individual nucleons: they can only exist in multiples of three. However, from the current theory of the strong interaction - quantum chromodynamics - arises the possibility that a phase transition may occur. If nuclear matter is sufficiently compressed that the nucleons begin to overlap - or the matter is heated sufficiently - a phase transition will occur. The quarks will become deconfined and a new state of matter will be formed: the quark-gluon plasma. There are three places wherein the quark-gluon plasma might be found:

1) in the milliseconds of the universe after the big bang

2) the interior of neutron stars:

3 ) in the heated volume of dense nuclear natter formed during the collision of relativistic heavy ions.

Clearly the first two of these are not readily accessible: to laboratory experiments. However, the third is currently the subject of intense and exciting experimental and theoretical efforts. The principal theoretisal tool used to describe the time evolution of the reactions is relativistic hydrodynamics.

The use of hydrodynamics to describe the collision of hadronic matter has a long history which dates from work of Fermi [1]. Pomeranchuk $[2]$ and Landau $[3]$ in the early fifties. They altempted to describe proton-protion scattering and the concomitant production of pions using statistical and hydrodynamical concepts. Their success encouraged other, later applications to different reactions, both at higher energies as well as for heavier. composite particles. Since this early work, the models have been refined and 
fresh concepts have been advanced utilizing new ideas from particle physics, quantum chromodynamics and other fields such as astrophysics. This article will very briefly review a few of the varied applications relativistic hydrodynamics has in the area of heavy ion reactions. A two-fluid model which overcomes certain of the limitations of the usual relativistic hydrodynamics in describing the physical processes and which also avoids the problems with causality associated with the introduction of dissipation into the hydrodynamic equations will be described. We refer the reader to the literature for more detailed descriptions of the application of hydrodynamics to heavy ion reactions [4,5] and hadron-hadron collisions [6,7].

At the relativistic velocities employed in the experiments, it is normal to measure mass, temperature and energy in units of energy as follows from the famous Einstein mass-energy equivalence relation $E=m c^{2}$. The standard unit of energy the electron volt or multiples of this such as a million electron volts which is abbreviated $\mathrm{MeV}$ and one thousand $\mathrm{MeV}$ or a GeV. The rest mass of a proton is approximately $939 \mathrm{MeV}$ or $0.939 \mathrm{GeV}$. Protons accelerated at CERN in Geneva have a kinetic energy of $200 \mathrm{GeV}$. Objects having a non-zero velocity relative to an observers rest-frame are contracted by an amount $\gamma=\left(1-\beta^{2}\right)^{-1 / 2}$ where $\beta=v / c$ and $c$ is the velocity of light. From simple relativistic expressions one has

$$
E=m c^{2}=T+m_{0} c^{2}=\gamma m_{0} c^{2}
$$

where $T$ is the kinetic energy and $m_{0}$ is the rest mass. Hence,

$$
\gamma=1+T / m_{0} \text {. }
$$

At these energies the Lorentz contraction factor $\gamma$ is 213 and the velocity is 0.999989 c. That is, the objects are 213 times larger in the transverse direction than in the longitudinal direction. Such severe distortions are difficult to inodel. However, because of Lorentz invariance the reaction can equally well be described in the center-of-mass frame in which each of the objects have an equal and opposite momentum. In this frame the two nucleons move towards cach other with a velocity of $0.9953 \mathrm{c}$ or a $\gamma$ of 10.4 .

It is not a priori apparent that hydrodynamics will te valid for the description of heavy ion reactions. An examination of the conditions necessary for the "alidity of hydrodynamics indicates that the requirements are only marginally fulfilled. For example, the number of particles which are involved in a heavy ion collision ranges from perhaps only 2 hundred to a thousand. Hence, the number of degrees of freedom is large compared to one. but relatively small compared to a usual fluid. If one creates a quark-gluon plasma Juring the collision, then the number of degrees of freedom will increase by at least a factor of three.

There is also the condition that there be sufficient time for the establishment of local, thermal equilibrium; this also is marginally satisfied. A lower limit on the collision time for two heavy ions may be roughly estimated as the nuclear diameter divided by the velocity of light, or about $5 \times 10.23 \mathrm{~s}$. Nucleons interact by exchanging pions and it requires about $5 \times 10^{-24} s$ for two adjacent nucleons to exchange a pion. Since this interaction time is about one-tenth of the total collision time. some degree of local equilibrium will be established. This will be particularly true for central ollisions of large nuclei for which the matter in the interior will be confined for longer periods than the above estimate. Further, for moderate bombarding energies, the actual 
reaction time is around $20 \times 10^{-23} \mathrm{~s}$ which is appreciably larger than the simple above argument suggested.

The question of whether there is local equilibrium has been investigated by many authors using a variety of numerical methods. Although there is time for only a very few collisions, because of the very strong nucleon-nucleon interaction, near-equilibrium conditions seem to prevail. A detailed computez simulation for the case of molecular collisions has been made by Bird [8] who concluded that only 2 - 3 collisions are needed to achieve thermal equilibrium. Bondorf and Zimányi [9] have investigated the approach to equilibrium using a time-dependent Boltzmann equation. They concluded that the pion and proton spectra suggesi that the momentum distributions are very near their equilibrium values. During sufficiently energiuc reactions, new particles such as pions or deltas may be created; such particles are short lived. either being rapidly absorbed in the case of pions, or decaying into a nucleon and a pion. Montvay and Zimányi [10] have investigated whether chemical equilibrium is reached; they conclude that it is not reached although the system is not very far from it.

Finally, it is not unrealistic to treat the nucleons as classical particles for the energy regimes in which we shall be interested. For relativistic nucleons their momenta is greater than $1 \mathrm{GeV} / \mathrm{c}$ and their corresponding de Broglie wavelength is $0.4 \mathrm{fm}$ (one fermi, $\mathrm{fm}$, is $10^{-13} \mathrm{~cm}$ ) which is less than the radius of a nucleon and much less ihan that of a nucleus.

The applications of relativistic fluid dynamics to heavy ion reactions have assuned there to be no dissipation. (There is some early work on hadronhadron reactions by the Russian school which attempted to include the effects of viscosity. This work assumed the validity of the Landau equations and is reviewed by Feinberg [6].) In the Los Alamos effort the thrue dimensional relativistic Euler equations are solved numerically using the particle-in-cell method developed by Harlow [11,12]. Since the PIC methor: is well-known and the differences required for the relativistic equations are treated in ref. 12 , the method of numerical solution will not be discussed here. The particle-incell method dllows calculations in cases of extreme distortion and shear including cases where cavities appear in the fluid. It also allows bertifu! graphical representations of the fluid (cf., ref. 5). However, it consumes vast amounts of computer memory.

Tile equation of state for nuclear matter is unknown: indeed, one of the goals of heavy ion reactions is to investigate the equation of state. Since theoretical calculations of the energy and pressure of nuclear $m a t t e r$ as a function of density and temperatureare quite uncertain, one usually is forsed to make assumptions regarding the equation of state. It is normal in nuclear physics to call the energy per nucleon, $E(n)$, the equation of state rather than expressing the pressure as a function of temperalure and density. The two are of course equivalent since one may obtain the pressure from the usual thermndynamic relation

$$
\left.P=\frac{\partial \mathbf{E}}{\partial V}\right)_{S},
$$

where $S$ is the entropy. Examples of some zero-temperature equations of states which have been used are shown in fig. 1 . One further usually assumes that 
the matter obeys a Fermi gas equation of state for non-zero temperature. For densities greater than five times normal nuclear matter density, a number of possible scenarios have been proposed. Currently, the most plausible one is that at sufficiently high densities or temperatures, the nucleons 'melt' and a quark-gluon plasma is formed in which the identities of the individual nucleons is lost and the constituent quarks and gluons are free to briefly roam about the relatively large collision volume.

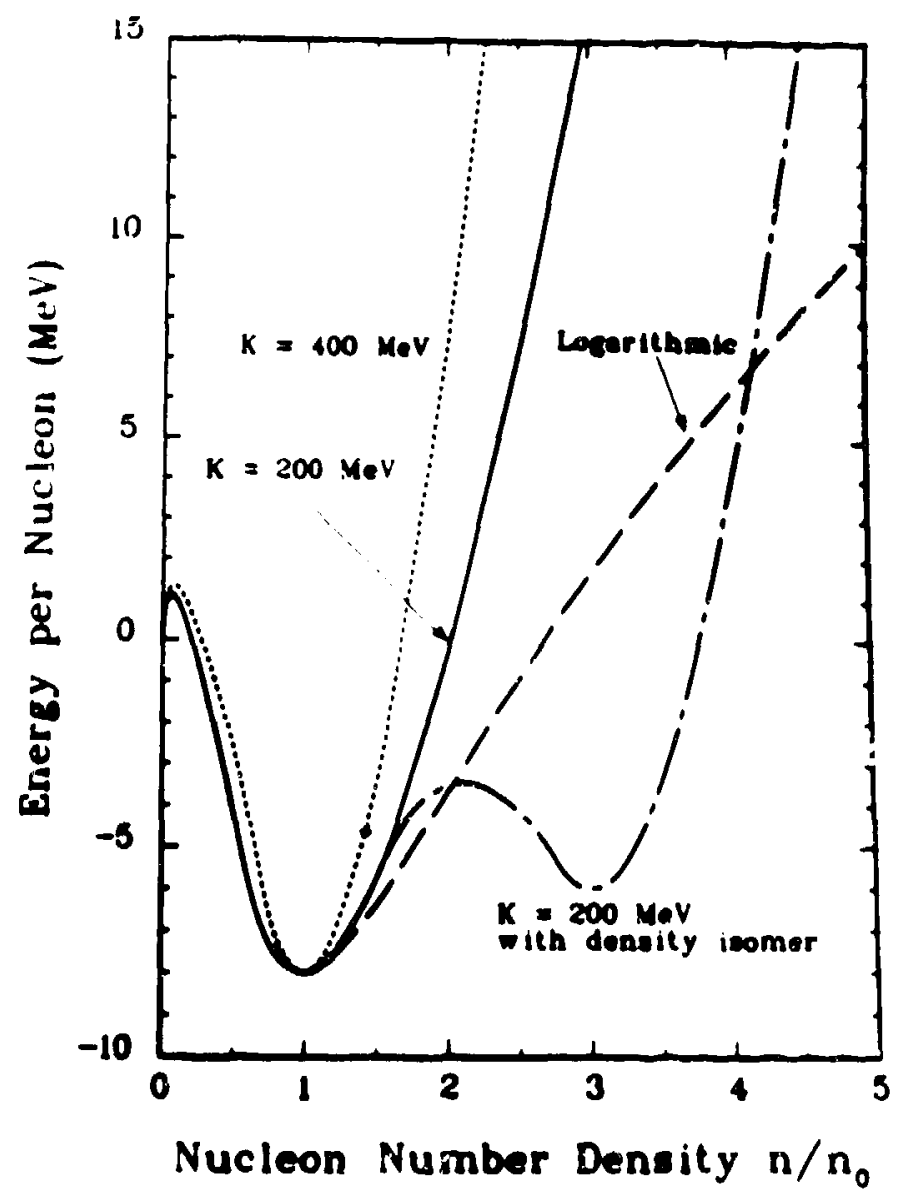

Fig. 1. A plot of the grcund state energy per nucleon $E(n)$ as a function of derisity for four different phenomenological expressions. The quantity $K$ is the compression modulus of nuclear matter. the canonical value of which is $200 \mathrm{McV}$.

An example of the time development of a hesvy ion reaction is shown in fig. 2 which shows the collision of two equal mass nuclei at $800 \mathrm{M}: \mathrm{V} /$ nucleon. From a knowledge of the velocity vectors of the Aluid in each cell at the end of the calculation, one may calculate the double differential cross section $d^{2} \sigma / d E$ d $\Omega$ which may be compared with experiment. In general the agriement is satisfactury. In fact hydrodynamic calculations predicted that in certain reactions, the relative incompressibility of nuclear mattet would cause the projectile to glance off the larget and produce a distinctive signal in the angular distribution. This "sideways" now was subsequently experimentally obseried. For details see ref. 4. 


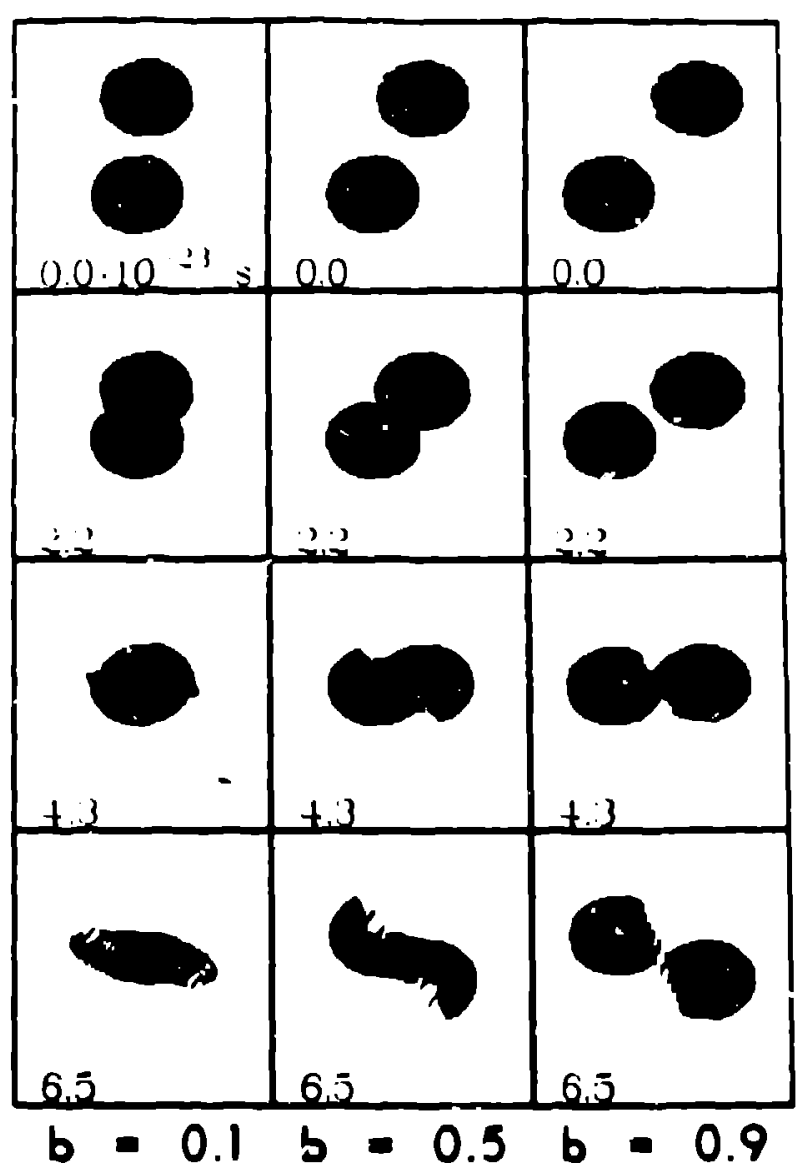

Fig. 2. Matter distributions for two equal-mass nuclei at $162 \mathrm{MeV} /$ nucleon in the center-of-miss (equivalent laboratory energy is 800 Mev/nucleon). Three impact parameters are shown in units of the sum of the radii of the two nuclei. Since the Euler equations are scale invariant, the figure applies to arbitrary mass nuclei, although the lime scale is appropriate only for $238 \mathrm{U}$ on $238 \mathrm{U}$.

A close examination of fig. 2 will demonstrates that the calculation reproduces the result that for a one-fluid system with no viscosity. the meanfree-path of the onatter is zero. (This is more easily seen when the matter from each nucleus is ploted in color us in ref. 5.) This is not a serious problem at low bombarding energies of less than GeV per nucleon for which the mean free path of nucleon ir the nucleus is much shorter than the nuclear diameter. However. as the energy of the projectile increases. the nucleus becomes more transpareat and the assumption of a zero mean-free-path becomes untenable. The eifect of non-zero mean-free-paths has been known for some time from high energy proton-nucleus experiments at Fermi Lab and CEKN: in these experiments the so-called leading particles punch through the largel and carry away, significat amount of the energy of the incident proton. One could simsizte to a small extent the effects of a non-zero meanfree-path by introducing viscosity. However, this would introduce all the 
problems associated with the acausal behaviour as demonstrated by Hiscock and Lindblom [13,14]. In any event, this would be inadequate when the meanfree-paths become so long that some of the nucleons can traverse the entire target and emerge on the far side. Further, as the energies increase it be comes less likely that local thermal equilibrium is instantaneously established at the interface of the two nuclei.

To describe the situation in which large mean-free-paths are involved, a two-fluid model was introduced [15]. To obtain the equations which describe the two-fluid model, each nucleus is assumed to be a fluid which has the identical properties of the fluid representing the other nucleus. When the two fluids ccllide iney are allowed to exchange energy and momentum at a finite rate proportional to the relative velocity of the two nuclei and to the nucleonnucleon cross section $\sigma_{\mathrm{NN}}$. Thus, the rate of momentum loss is finite and the two fluids will interpenetrate. The amount of interpenetration is small at low energies for which $\sigma_{N N}$. is large and increases as $\sigma_{N N}$ decreases. The Euler equations which ensure particle number conservation remain unchanged, but the equations ensuring energy and momentum conservation must be riodified to allow an interchange of these quantities. The changes are in the form of dditional terms, the magnitude of which can be estimated from kinetic theory: if one knows the collision rate and the amount of energy and momentun lost in each collision, then the total amount of loss may be found.

The expression for the collision rate is

$$
R_{\text {coll }}=N_{1} N_{2} \sigma_{\mathrm{NN}} \quad v_{\text {rel }}
$$

where $N_{1}$ and $N_{2}$ are the densities of the two fluids and $v_{r e l}$ is the relativistic generalizaticn of the relative velocity. The generalized Euler equations for fluid one are

$$
\begin{aligned}
& \partial_{t} M_{1}+\nabla\left(v_{1} \cdot M_{1}\right)=-\nabla P-R_{\text {coll }} K\left(\gamma_{1} v_{1}-\gamma_{2} v_{2}\right) / Y \\
& \partial_{t} E_{i}+\nabla\left(v_{1} E_{1}\right)=-\nabla P-R_{\text {coll }} K\left(\gamma_{1}-\gamma_{2}\right) / Y
\end{aligned}
$$

where $M_{1}$ and $E_{1}$ are the momentum and energy densities of fluid one and $Y$ is the scalar product of the two four-velocities

$$
\mathbf{Y}=\left(\mathbf{u}_{1} \mathbf{u}_{2}\right) \text {. }
$$

The quantity $K$ determines the amount of energy-momentum ioss and is fixed by comparing with high energy nucleon-nucleus reactions. The equations for fluid two are obtained by interchanging the indices 1 and 2 .

Unlike the Euler equations, eqs (2) and (3) are not scale invariant; lite calculated results will depend the masses of the nuclei involved which is entirely reasonable. A similar consequence occurs if one uses the NavierStokes equations. However, unlike the case of the Navier-Stokes equations which introduces dissipation through higher order derivatives of the velocity. the two-fluid model partially achieves the same result by eliminating derivatives in the additional terms.

The additional coupling terms in eqs. (2) and (3) describe the friction between the two nuclei entirely in terms of two-body collisions of the constituent nucleoss. It is assumed that the nucleon-nucleon cross section is the free $N N$ cross section $\sigma_{N N}$ and is independent of density and temperature: this assumption is surely poor at high temperatures and densities. It is further 
assumed that the Fermi velocities of the nucleons may be ignored. For large relative velocities this is a good approximation (the Fermi velocity at nomal nuclear density is approximately $0.27 \mathrm{c}$ ); for lower bombarding energies, one must worry about the effects due to the Fermi velocity. For methods which partially take into account the effects of the Fermi velocity, the reader is referred to refs. 5 and 15 . In addition both the one-fluid and two-fluid models necessarily omit binding energy effects.

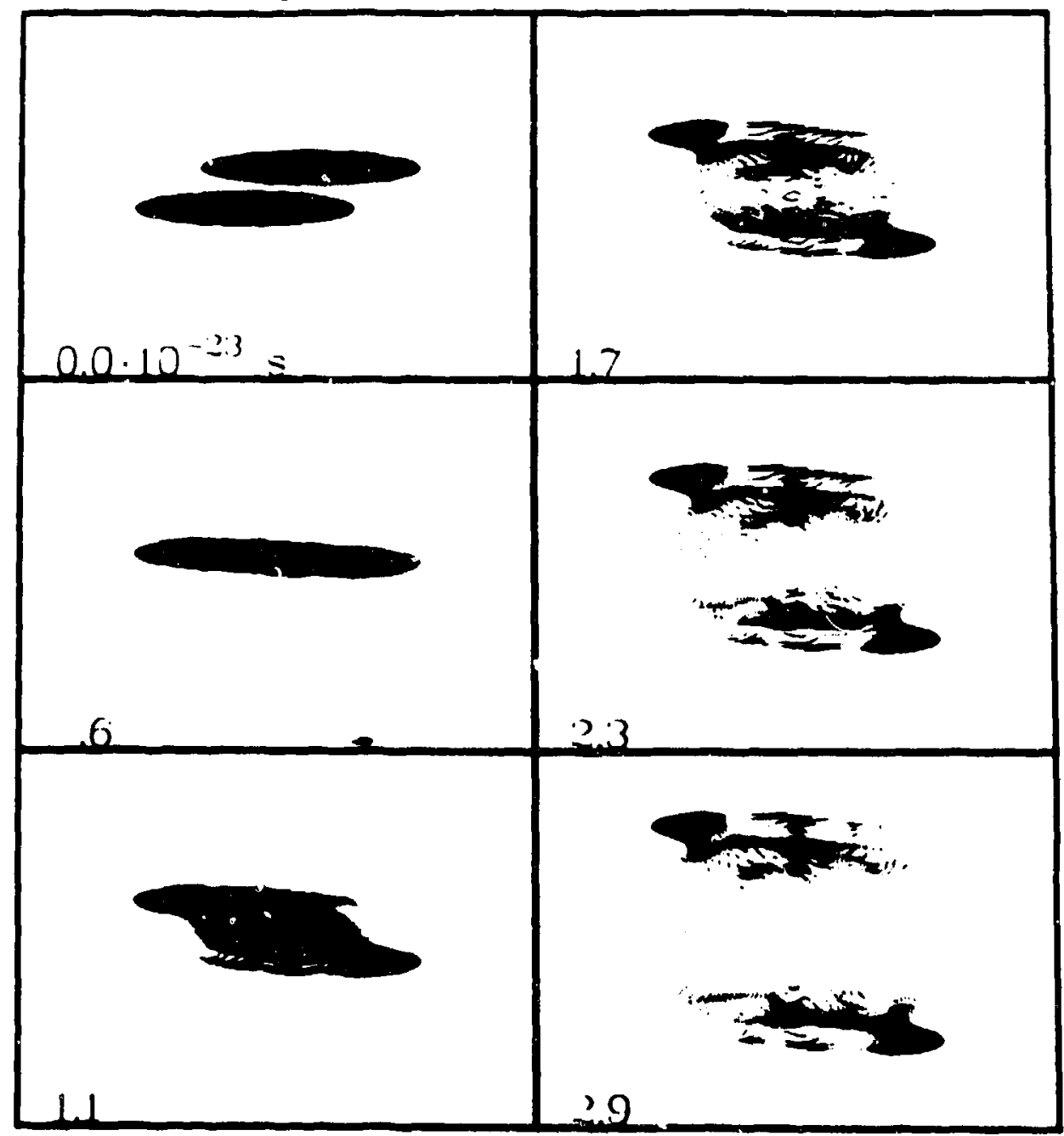

Fig. 3. Matter distributions for $238 \mathrm{U}$ on $238 \mathrm{U}$ calculated in the center-of-mass system with the two-fluid model. The center-of-mass kinetic energy is 5 GeV/nucleon (equivalent laboratory energy is $73.7 \mathrm{GeV} /$ nucleon) and the impact parameter is 0.3 .

In figure 3 resulis are shown for a collision between two equal $m$ a s $s$ nuclei, each having an energy of $3 \mathrm{GeV}$ per nucleon in the center of mass frame. (This energy corresponds to a velocity of $0.987 \mathrm{c}$ in the center of mass or a velocity oi $0.99992 \mathrm{c}$ in the laboratory frame.) The effects of a non-zero mean-free-path are immediately evident. The two nuclei essentially pass 
through each other, although each nucleus exerts a drag upon the other. in the one-fluid model the matter at the interface of the two nuclei would have come to a halt. All the kinetic energy must be converted into thermal energy. Hence, the one-fluid model can expect to exhibit a larger thermal pressure than does the two-fiuid model. This will result in the nuclear matter blowing up and disintegrating sooner.

Experiments have recently begun at CERN which collide 200 GeV/nucleon 160 ions on nuclear targets in a search for signals of a quarkgluon plasma. Similar experiments will soo 1 begin at lower energies at Brookhaven National Laboratory. In all these experiments relativistic hydrodynamics will play an essential role in the interpretation of results.

\section{References}

1. E. Ferm:, Prog. Theor. Phys. 5(1950)570.

2. I.U. Pomeranchuk, Dokl. Akad. Nauk. USSR 78(1951)889.

3. L. D. Landau, Izv. Akad. Nauk. SSSR Sci Fiz. 17(1953)51.

4. H. Stocker and W. Greiner, Phys. Rept. 137(1986)277.

5. R.B. Clare and D. Strottman, Phys. Rept. 141(1986)177.

6. E.L. Feinberg, Phys. Rept. 5(1972)237.

7. E.V. Shuryak, Phys. Rept 61(1980)71.

8. G.A. Bird, Phys. Fluids 6(1963) 1518 .

9. J. Bondorf and J. Zimányi, Phys. Scri. 24(1981)758.

10. I. Montvay and J. Zimányi, Nucl. Phys. A316(1979)490.

11 F.H. Harlow, Los Alamos Scientific Report, LAMS-1956(1955).

12. F.H. Harlow, A.A. Amsden and J.R. Nix, J. Comp. Phys. 20(1976)119.

13. W.A. Hiscock and L. Lirdblom, Ann. Phys. (NY) 151(1983)466.

14. W.A. Hiscock and L. Lindblom, Phys. Rev. $31 \mathrm{D}(1985) 725$.

15. A.A. Amsden, A.S. Goldhaber, F.H. Harlow and J.R. Nix, Phys. Rev. C27(1978)2080. 